\title{
Comparison of Dye Adsorption of Three Forms of Chitosan
}

\author{
Shu-Hwa Lin ${ }^{*}$, Chih-Pong Chang' ${ }^{2}$, Jen-Chieh Chang ${ }^{3}$, Kelly Mammel ${ }^{1}$ \\ ${ }^{1}$ Department of Family \& Consumer Sciences, University of Hawaii at Manoa, Honolulu, USA \\ ${ }^{2}$ Department of Textile Engineering, Chinese Culture University, Taipei, Taiwan \\ ${ }^{3}$ Institute of Materials Science and Nanotechnology, Chinese Culture University, Taipei, Taiwan \\ Email: shulin@hawaii.edu
}

Received 21 May 2014; revised 15 June 2014; accepted 30 June 2014

Copyright (C) 2014 by authors and Scientific Research Publishing Inc.

This work is licensed under the Creative Commons Attribution International License (CC BY). http://creativecommons.org/licenses/by/4.0/

(c) (i) Open Access

\section{Abstract}

The purpose of this project is to explore the adsorption rates of different dye methods (direct, reactive, acidic, disperse, azo dyes) in three shapes of chitosan materials (film, microsphere, and scaffold) which were formed by $\mathbf{8 8 \%}$ deacetylation degree of chitosan. Results of this research suggest that the samples with larger specific surface areas tend to have shorter adsorption times. Among the three samples, the chitosan scaffold reached the adsorptive saturation on the third day, showing the shortest adsorption time of the direct, reactive, acidic, dispersive and azo dyes, whereas the chitosan film reached the adsorptive saturation on the eighth day, giving it the longest adsorption time of the dyes.

\section{Keywords}

Chitosan, Film, Microsphere, Scaffold, Dye Adsorption

\section{Introduction}

Chitin, polysaccharide-based biopolymer, is a derived form of glucose which makes up most of the cell walls of fungi, the exoskeletons of arthropods, and on the sponge cucumber of the plant kingdom. Also, chitin can be deacetylated by exposure to concentrated alkali at high temperature to generate a polysaccharide called chitosan, which can polymerize into cellulose-like structure. The positive charged amino group of chitosan enables a lot of chemical reactions, including chelating of metal ions, cross-linking, chemical adsorption, etc. Thus, many researchers have been focusing on chitosan for a variety of different applications and studies.

Chitosan demonstrates high adsorption and performs as carrier when exposed to other materials, such as dyes, heavy metals, protein [1]-[6]. The excellent adsorptive property of chitosan is due to the presence of a hydroxyl

${ }^{*}$ Corresponding author. 
group $(-\mathrm{OH})$ and an amino group $\left(-\mathrm{NH}_{2}\right)$, which can form coordinate bond with the anion of heavy metal to generate heavy metal ion complex. In addition, chitosan is bio-degradable and environmental friendly [7]-[10]. The purpose of this project is to explore the adsorption rates of different dye methods (direct, reactive, acidic, disperse, azo dyes) in three shapes of deacetylated chitosan materials (film, microsphere, and scaffold) which were formed by $88 \%$ of deacetylated chitosan.

Many carcinogenic materials found in the environment contain benzene ring structure. Especially, dye materials have benzene rings which could possibly be carcinogenic as well. Thus, it is important and necessary to search for an effective way to purify sewage (particularly for sewage containing dyes) [11]. Research involving heavy metals and dye adsorption from sewage was not popular before. Until recently, the International Cancer Research Center has proven that untreated dyes contain benzene ring chemical, which can enter the food chain from shrimp and fish to human. Failed to be metabolized, this chemical can accumulate in human body and may eventually become cytopathogenic [12].

Researchers have found that chitosan can adsorb many materials special dye molecules [1]-[5]. However, among the relevant studies, the effects of different degrees of deacetylation of chitosan on the adsorption of specific dyes, or the formula of using fixed chitosan materials for the adsorption of a single dye have not been investigated [13]-[15]. Moreover, there are very little studies focusing on the different forms of chitosan materials or the adsorptive properties of different types of dyes. Therefore, this project was to explore the adsorptive properties of different dyes (i.e., direct, reactive, acidic, disperse, azo dyes) in three shapes of deacetylated chitosan materials (i.e., film, microsphere, and scaffold) which were formed by $88 \%$ of deacetylated chitosan. The expected result would be to find a simple and effective method to eliminate the carcinogenic dye materials from the sewage.

\section{Experiment}

\subsection{Preparation}

\subsubsection{Preparation of Chitosan Film Adsorbent}

The powder of $88 \%$ deacetylated chitosan (ACROS, ORGANICS) was dissolved into 1\% acetic acid to form 3\% chitosan colloid. In a $2 \mathrm{~cm} \times 2 \mathrm{~cm}$ square container, $2 \mathrm{~mL}$ of $3 \%$ chitosan colloid was poured and was placed into an oven (OVEN 453, HSIANGTAI CO., Taiwan) to dehydrate for 24 hours to form a chitosan film. The chitosan film was solidified by slowly stirring in $1 \%$ sodium hydroxide for 30 minutes by using a magnetic stirrer (SP18425, Apogent Technologies). After drying at $30^{\circ} \mathrm{C}$, the chitosan film with a thickness of about $0.3 \mathrm{~mm}$ can be stored at $25^{\circ} \mathrm{C}$ and $65 \% \mathrm{RH}$ in a programmed temperature and humidity chamber.

\subsubsection{Preparation of Chitosan Microsphere Adsorbent}

By using a syringe, $2 \mathrm{~mL}$ of $3 \%$ chitosan colloid was dropped slowly into 1\% sodium hydroxide and was solidified for 30 minutes. The solidified chitosan microsphere was then washed with deionized water and was dried at $30^{\circ} \mathrm{C}$ in an oven. The dried chitosan microsphere was stored at $25^{\circ} \mathrm{C}$ and $65 \% \mathrm{RH}$ in a programmed temperature and humidity chamber. Measured by the MASTERSIZER, a Laser Diameter Analyzer (Hydro 2000SM, Malvern Instrument, UK), the size of each chitosan microsphere was about $1.2 \pm 0.3 \mathrm{~mm}$.

\subsubsection{Preparation of Chitosan Scaffold Adsorbent}

The 3\% chitosan colloid was pumped with air to form bubbles and was homogenized at 30,000 rpm by using a high speed homogenizer (HG-300D, HSIANGTAI CO., Taiwan). In a container with a diameter of $1.5 \mathrm{~cm}$ and a height of $2 \mathrm{~cm}, 2 \mathrm{~mL}$ of the $3 \%$ chitosan colloid was dropped to fix the shape at low temperature, followed by solidifying in $1 \%$ sodium hydroxide for 30 minutes. The solidified chitosan scaffold absorbent was washed with deionized water and was dried by using a freeze dryer (SMR-100, FUYUAN CO., Taiwan). The dried chitosan scaffold was stored at $25^{\circ} \mathrm{C}$ and $65 \% \mathrm{RH}$ in a programmed temperature and humidity chamber. A SEM picture of the chitosan scaffold was shown in Figure 1 (6335F, JEOl, Japan).

\subsection{Types of Dyes and the Evaluation of the Adsorptive Rates}

\subsubsection{Analysis of the Properties of Dyes}

In this experiment, five different dye samples (i.e., direct, reactive, acidic, disperse, azo dyes) were tested. In 


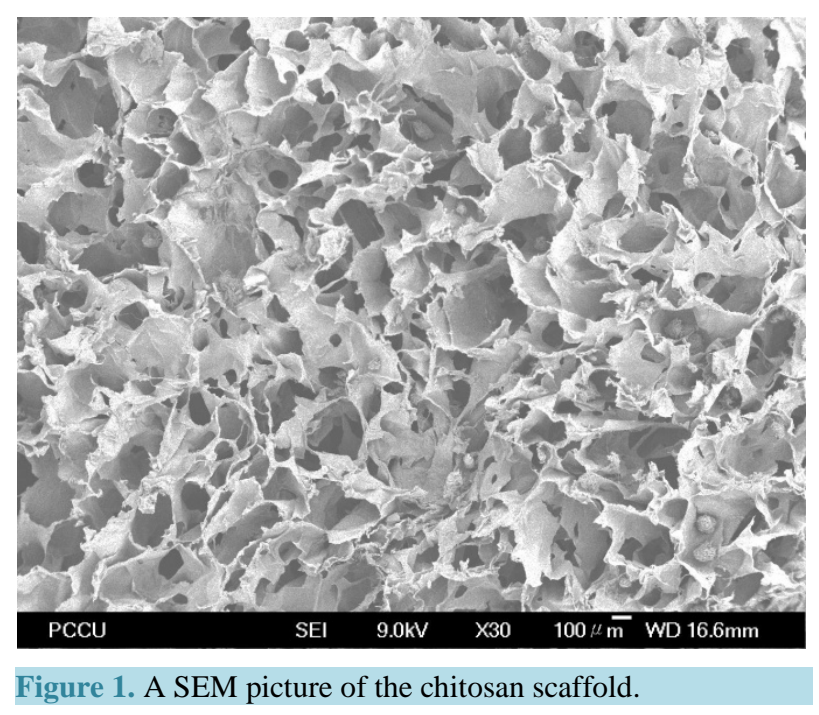

Table 1, the chemical structures of the dyes were shown. Except for the azo dye, which was synthesized in the lab, all the other dyes were provided by the Plastoform Industries Ltd (Taiwan). About 10 mg of each dye was used to form a corresponding dye solution with a concentration of $0.1 \mathrm{mg} / \mathrm{mL}$. About $2 \mathrm{~mL}$ of each dye solution was poured into a quartz test tube and was placed into a photospectrometer (V-670, JASCO). The wavelength was increased by $1 \mathrm{~nm}$ each time from 350 to $760 \mathrm{~nm}$. The light absorbances of each dye solution at different wavelengths were recorded. A standard curve was formed for each dye solution according to the greatest absorbance and the corresponding wavelength. For a single dye, five different concentrations were prepared and $2 \mathrm{~mL}$ of each concentration was then poured into a quartz test tube to measure the absorbencies. According to the absorbance of different concentrations, a formula of the standard curve of each dye solution was formed. The greatest wavelength, the formula of the standard curve, and the $\mathrm{R}^{2}$ value of each dye solution were shown in Table 2.

\subsubsection{Evaluation of the Adsorptive Rates}

Three different forms of chitosan samples (i.e. film, microsphere and scaffold) were put into $0.05 \mathrm{~g}$ of each dye solution, respectively. The adsorptive properties of the samples were recorded daily and the time for saturated adsorption was noted for each sample. The chitosan sample with saturated adsorption was dissolved into 5\% acetic acid. Using 5\% acetic acid as the standard solution, the adsorbencies of the samples were recorded. The adsorptive rate of each sample can be calculated by using the formula:

$$
C(\%)=\left(C_{1} / C_{2}\right) \times 100
$$

$C_{1}$ is the adsorbance difference between the chitosan sample before dyeing and the chitosan sample dissolved into $5 \%$ acetic acid after dyeing.

$C_{2}$ is the adsorbance difference between the purified water and the dye solution.

\section{Results and Discussion}

\subsection{The Adsorptive Properties of Different Forms of Chitosan to the Disperse Dye}

The adsorptive curves of different forms of chitosan adsorbed with the disperse dye (C. I. Disperse Red 82) for 10 days at a fixed temperature was shown in Figure 2. The time for saturated adsorption by the chitosan film was 8 days and the adsorptive rate was 55.7\%; the time for saturated adsorption by the chitosan microsphere was shortened to 6 days and the adsorptive rate was increased to $60.6 \%$; the time for saturated adsorption by the chitosan scaffold was further decreased to 4 days and the adsorptive rate was increased to $65.9 \%$. Hence, the time for saturated adsorption and the adsorptive rate for the reactive dye were changed according to the different forms of chitosan samples. The experimental results indicated that the forms of chitosan, from film to scaffold, could shorten the time for saturated adsorption from 8 days to 4 days, and could increase the adsorptive rate by 


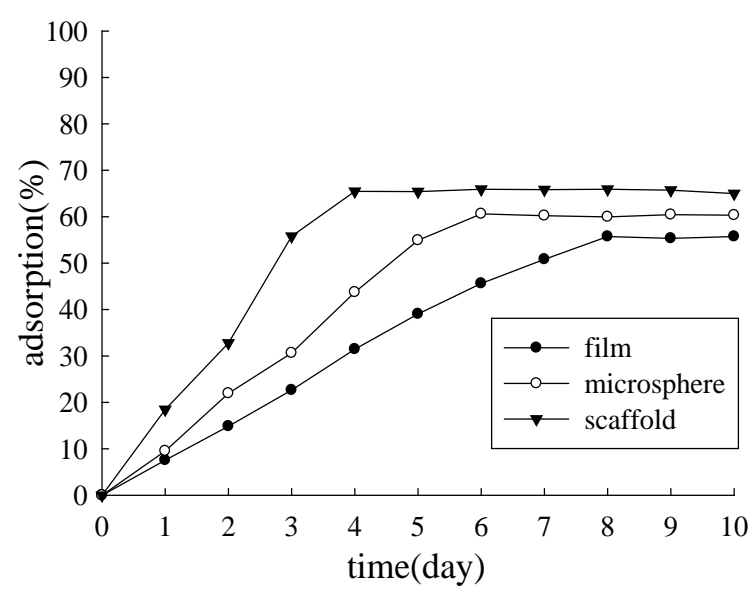

Figure 2. The adsorptive curves of different forms of chitosan samples adsorbed with the disperse dye (i.e. C. I. Disperse Red 82) at a fixed temperature.

Table 1. The basic information of each dye.

C. I. Disperse Red 82

*Azo dye was synthesized and was provided by Prof. Lin, one of the authors of this paper.

Table 2. The formulas and the $\mathrm{R}^{2}$ values of standard curves of the dyes.

\begin{tabular}{cccc}
\hline Name of Dye & Absorptive Wavelength (nm) & Formula of the Standard Curve & $\mathrm{R}^{2}$ Value \\
\hline C. I. Disperse Red 82 & $626 \mathrm{~nm}$ & $\mathrm{y}=0.258 \mathrm{x}$ & 0.971 \\
C. I. Direct Blue 185 & $552 \mathrm{~nm}$ & $\mathrm{y}=0.449 \mathrm{x}$ & 0.985 \\
C. I. Acid Blue 193 & $577 \mathrm{~nm}$ & $\mathrm{y}=0.355 \mathrm{x}$ & 0.985 \\
C. I. Reactive Yellow 145 & $419 \mathrm{~nm}$ & $\mathrm{y}=0.351 \mathrm{x}$ & 0.940 \\
New Azo Dye & $484 \mathrm{~nm}$ & $\mathrm{y}=0.423 \mathrm{x}$ & 0.986 \\
\hline
\end{tabular}


about $10.4 \%$ from $55.5 \%$ to $65.9 \%$. These results were due to the differences of the surface areas of different forms of chitosan samples. According to the adsorptive curve in Figure 2, the scaffold had the highest surface area, followed by the microsphere; the film had the least surface area. The adsorptive rate increased with the corresponding increase of the surface area, from 8 days to 4 days.

\subsection{The Adsorptive Properties of Different Forms of Chitosan to the Direct Dye}

The adsorptive curves of different forms of chitosan absorbed with the direct dye (C. I. Direct Blue 185) for 10 days at a fixed temperature was shown in Figure 3. The time for saturated adsorption by the chitosan film was 8 days and the adsorptive rate was $90.9 \%$; the time for saturated adsorption by the chitosan microsphere was shortened to 5 days and the adsorptive rate was increased to 92.6\%; the time for saturated adsorption by the chitosan scaffold was further decreased to 3 days and the adsorptive rate was increased to $94.9 \%$. The experimental results showed that the forms of chitosan, from film to scaffold, could shorten the time for saturated adsorption from 8 days to 3 days, and could increase the adsorptive rate by about $4.0 \%$ from $90.9 \%$ to $94.9 \%$.

\subsection{The Adsorptive Properties of Different Forms of Chitosan to the Acidic Dye}

The adsorptive curves of different forms of chitosan absorbed with the acidic dye (C. I. Acid Blue 193) for 10 days at a fixed temperature was shown in Figure 4. The time for saturated adsorption by the chitosan film was 8 days and the adsorptive rate was $78.5 \%$; the time for saturated adsorption by the chitosan microsphere was

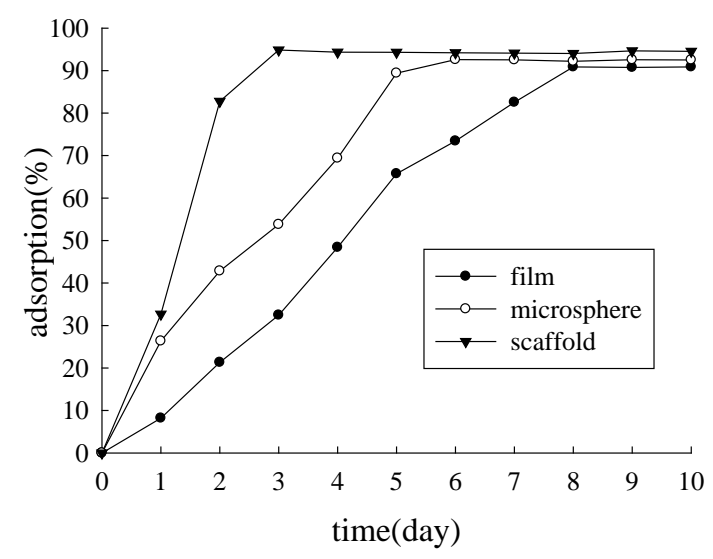

Figure 3. The adsorptive curves of different forms of chitosan samples adsorbed with the direct dye (i.e. C. I. Direct Blue 185) at a fixed temperature.

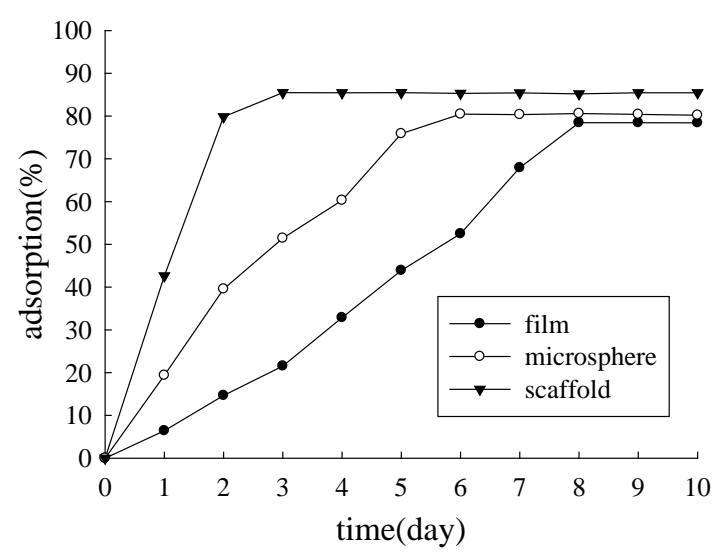

Figure 4. The adsorptive curves of different forms of chitosan samples adsorbed with the acidic dye (i.e. C. I. Acid Blue 193) at a fixed temperature. 
shortened to 5 days and the adsorptive rate was increased to 80.6\%; the time for saturated adsorption by the chitosan scaffold was further decreased to 3 days and the adsorptive rate was increased to $85.5 \%$. The experimental results showed that the forms of chitosan, from film to scaffold, could shorten the time for saturated adsorption from 8 days to 3 days, and could increase the adsorptive rate by about $7.0 \%$ from $78.5 \%$ to $85.5 \%$.

\subsection{The Adsorptive Properties of Different Forms of Chitosan to the Reactive Dye}

The adsorptive curves of different forms of chitosan adsorbed with the reactive dye (C. I. Reactive Yellow 145) for 10 days at a fixed temperature was shown in Figure 5. The time for saturated adsorption by the chitosan film was 8 days and the adsorptive rate was $91.6 \%$; the time for saturated adsorption by the chitosan microsphere was shortened to 5 days and the adsorptive rate was increased to 92.9\%; the time for saturated adsorption by the chitosan scaffold was further decreased to 3 days and the adsorptive rate was increased to 95.9\%. The experimental results showed that the forms of chitosan, from film to scaffold, could shorten the time for saturated adsorption from 8 days to 3 days, and could increase the adsorptive rate by about $4.3 \%$ from $91.6 \%$ to $95.9 \%$.

\subsection{The Adsorptive Properties of Different Forms of Chitosan to the Azo Dye}

The adsorptive curves of different forms of chitosan adsorbed with the New Azo dye for 10 days at a fixed temperature was shown in Figure 6. The time for saturated adsorption by the chitosan film was 8 days and the adsorptive rate was $85.9 \%$; the time for saturated adsorption by the chitosan microsphere was shortened to 5 days

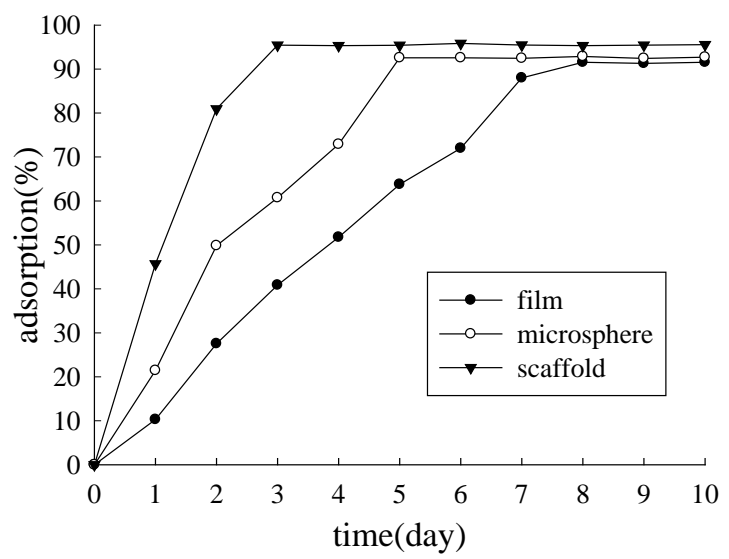

Figure 5. The adsorptive curves of different forms of chitosan samples adsorbed with the reactive dye (i.e. C. I. Reactive Yellow 145) at a fixed temperature.

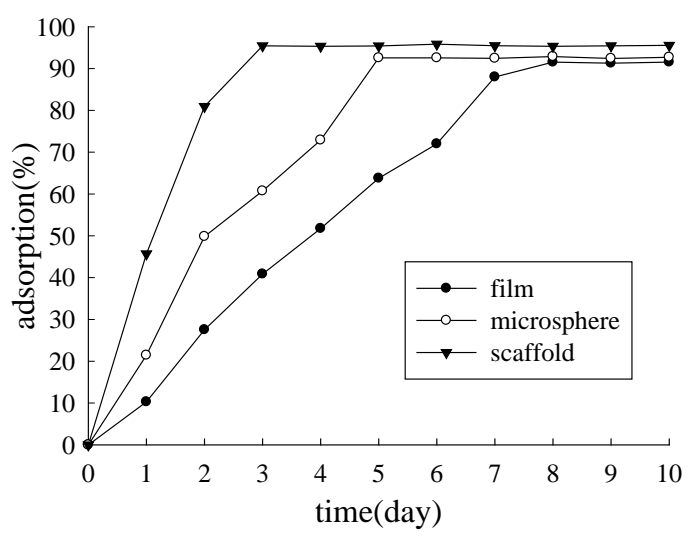

Figure 6. The adsorptive curves of different forms of chitosan samples adsorbed with the azo dye at a fixed temperature. 
and the adsorptive rate was increased to $89.7 \%$; the time for saturated adsorption by the chitosan scaffold was further decreased to 3 days and the adsorptive rate was increased to $92.9 \%$. The experimental results showed that the forms of chitosan, from film to scaffold, could shorten the time for saturated adsorption from 8 days to 3 days, and could increase the adsorptive rate by about $7.0 \%$ from $85.9 \%$ to $92.9 \%$.

\subsection{Analysis of the Adsorptive Properties of Chitosan Scaffolds in Different Dyes at a Fixed Temperature}

According to the Figures 2-6, due to the largest surface area, chitosan scaffold had the best results on the time for saturated adsorption and the adsorptive rate. Hence, chitosan scaffold was used in this experiment to investigate its adsorptive properties with different dyes. The adsorptive curves of chitosan scaffold adsorbed with different dyes were shown in Figure 7. The time required for saturated adsorption of the direct (C. I. Direct Blue 185), acidic (C. I. Acid Blue 193), reactive (C. I. Reactive Yellow 145) and New Azo dyes by chitosan scaffold were all 3 days; whereas the time required for saturated adsorption of the disperse dye (C. I. Disperse Red 82) was 4 days. Chitosan scaffold showed different adsorptive rates with different dyes. Chitosan scaffold with the reactive dye (C. I. Reactive Yellow 145) showed the best adsorptive rate (95.9\%), followed by the direct (C. I. Direct Blue 185), New Azo, and acidic dyes (C. I. Acid Blue 193) with the adsorptive rates of $94.9 \%, 92.9 \%$ and 85.5\%, respectively; chitosan scaffold with the disperse dye (C. I. Disperse Red 82) showed the worst adsorptive rate of $65.9 \%$.

The above results were caused by the different chemical structures of different dyes. Based on the chemical structures, the dye with more $\left(-\mathrm{SO}_{3}^{-}\right)$tended to has better adsorptive result. The dye adsorptive property of chitosan was due to the presence of the amino group of the six membered ring of chitosan. The amino group, changing from $-\mathrm{NH}_{2}$ to $-\mathrm{NH}_{3}^{+}$in dye solution, can form ionic bond with sulfonate ( $-\mathrm{SO}_{3}^{-}$) of the dye easily. The reactive dye (C. I. Reactive Yellow 145) with the greatest number of sulfonate $\left(-\mathrm{SO}_{3}^{-}\right)$led to the best adsorptive results; whereas the disperse dye (C. I. Disperse Red 82) with no sulfonate ( $\left.-\mathrm{SO}_{3}^{-}\right)$showed the worst adsorptive results.

\section{Conclusion}

This project was to investigate the adsorptive properties of three different forms of chitosan (i.e. film, microsphere and scaffold) with the five different dyes, including direct (C. I. Direct Blue 185), reactive (C. I. Reactive Yellow 145), acidic (C. I. Acid Blue 193), disperse (C. I. Disperse Red 82), and New Azo dyes. The experimental results indicated that chitosan scaffold had the best results of the time for saturated adsorption and the adsorptive rate with the five selected dyes, followed by the chitosan microsphere; the chitosan film samples had the worst results. The chitosan scaffold sample significantly shortened the time for saturated adsorption from 8 days to 3 - 4 days; it also increased the adsorptive rates from $4.0 \%$ with the direct dye to $10.4 \%$ with the disperse dye. Comparing the adsorptive properties of chitosan scaffold sample to different types of dyes, the reactive dye (C. I. Reactive Yellow 145) showed the best adsorptive rate of 95.5\%, followed by the direct (C. I. Direct Blue

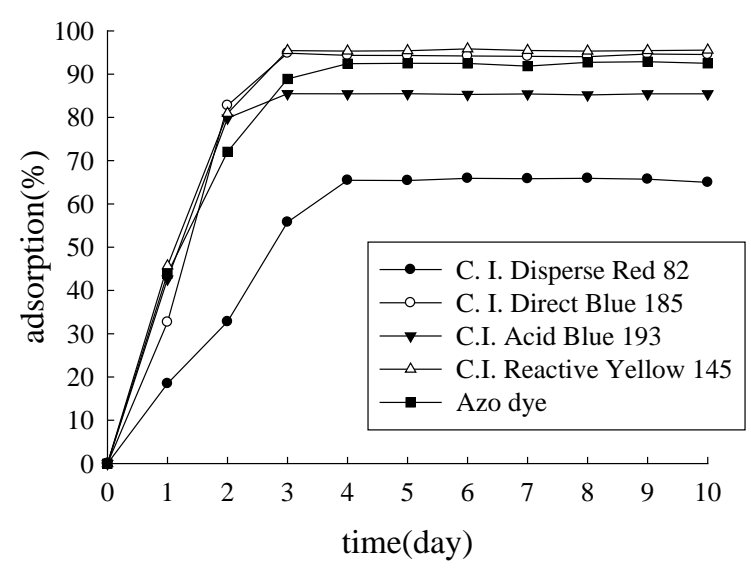

Figure 7. The adsorptive curves of chitosan scaffold sample adsorbed with different dyes at a fixed temperature. 
185) and New Azo dyes with the adsorptive rates of $94.9 \%$ and $92.9 \%$, respectively; the acidic dye (C. I. Acid Blue 193) had adsorptive rate of 85.5\%; the disperse dye (C. I. Disperse Red 82) had the worst adsorptive rate of $65.9 \%$.

\section{Acknowledgements}

The authors are grateful for the research financial partially support from the research support administered by the College of Tropical Agriculture and Human Resources, University of Hawaii at Manoa.

\section{References}

[1] Grini, G. and Giangiacomo, T. (2008) Absorption of C. I. Basic Blur 9 on Chitosan-Based Materials. International Journal of Environment and Pollution, 34, 451-465. http://dx.doi.org/10.1504/IJEP.2008.020809

[2] Minamisawa, H., Iwanami, H., Arai, N. and Okutani, T. (1999) Absorption Behavior of Cobalt(II) on Chitosan and Its Determination by Tungsten Metal Furnaceatomic Absorption Spectrometry. Analytica Chimica Acta, 378, 279-285. http://dx.doi.org/10.1016/S0003-2670(98)00641-2

[3] Kawamura, Y., Yoshida, H., Asai, S. and Tanibe, H. (1997) Breakthrough Curve for Adsorption of Mercury (II) on Polyminated Highly Porous Chitosan Beads. Water Science and Technology, 35, 97-105. http://dx.doi.org/10.1016/S0273-1223(97)00119-4

[4] Chu, K.H. (2002) Removal of Copper from Aqueous Solution by Chitosan in Prawn Shell: Adsorption Equilibrium and Kinetics. Journal of Hazardous Materials, B90, 77-95. http://dx.doi.org/10.1016/S0304-3894(01)00332-6

[5] Liu, X., Tokura, S., Haruki, M., Nishi, N. and Sakairi, N. (2002) Surface Modification of Nonporous Glass Beads with Chitosan and Their Adsorption Property for Transition Metal Ions. Carbohydrate Polymers, 49, 103-108. http://dx.doi.org/10.1016/S0144-8617(01)00308-3

[6] Gan, Q. and Wang, T. (2007) Chitosan Nanoparticle as Protein Delivery Carrier-Systematic Examination of Fabrication Conditions for Efficient Loading and Release. Colloids and Surfaces Biointerfaces, 59, 24-34. http://dx.doi.org/10.1016/j.colsurfb.2007.04.009

[7] Wang, W., Du, Y. and Wang, X. (2008) Physical Properties of Fungal Chitosa. World Journal of Microbiology and Biotechnology, 24, 2717-2720. http://dx.doi.org/10.1007/s11274-008-9755-x

[8] Singla, A.K. and Chawla, M. (2001) Chitosan: Some Pharmaceutical and Biological Aspects-An Update. Journal of Pharmacology and Pharmacotherapeutics, 53, 1047-1067. http://dx.doi.org/10.1211/0022357011776441

[9] Shahidi, F., Arachchi, J.K.V. and Jeon, Y.J. (1999) Food Applications of Chitin and Chitosan. Trends in Food Science Technology, 10, 37-51. http://dx.doi.org/10.1016/S0924-2244(99)00017-5

[10] Arthur, P.A. and Panda, T. (1999) Studies on Applications of Chitin and Its Derivatives. Bioprocess Engineering, 20, 505-512. http://dx.doi.org/10.1007/s004490050622

[11] Chan, Y.F. (2001) The Production, Evaluation and Applications of Chitin and Chitosan. National Science Council Monthly, 29, 776-787.

[12] Ko, F.C., Cheng, J.O. and Wang, W.H. (2006) Critical Pathways of Bioaccumulation in Aquatic Food Webs: A Review. Platax, 4, 43-67.

[13] Uthairatanakij, A., Da Silva, J. and Obsuwan, K. (2007) Chitosan for Improving Orchid Production and Quality. Orchid Science and Biotechnology. Global Science Books, 1, 1-5.

[14] Shimizu, Y., Dohmyou, M., Yoshikawa, M. and Takagishi, T. (2004) Dyeing Chitin/Cellulose Composite Fibers with Reactive Dyes. Textile Research Journal, 74, 34-38. http://dx.doi.org/10.1177/004051750407400106

[15] Shimizu, Y., Dohmyou, M., Yoshikawa, M. and Takagishi, T. (2002) Dyeing Chitin/Cellulose Composite Fibers with Acid Dyes. Textile Research Journal, 72, 563-567. http://dx.doi.org/10.1177/004051750207200701 
Scientific Research Publishing (SCIRP) is one of the largest Open Access journal publishers. It is currently publishing more than 200 open access, online, peer-reviewed journals covering a wide range of academic disciplines. SCIRP serves the worldwide academic communities and contributes to the progress and application of science with its publication.

Other selected journals from SCIRP are listed as below. Submit your manuscript to us via either submit@scirp.org or Online Submission Portal.
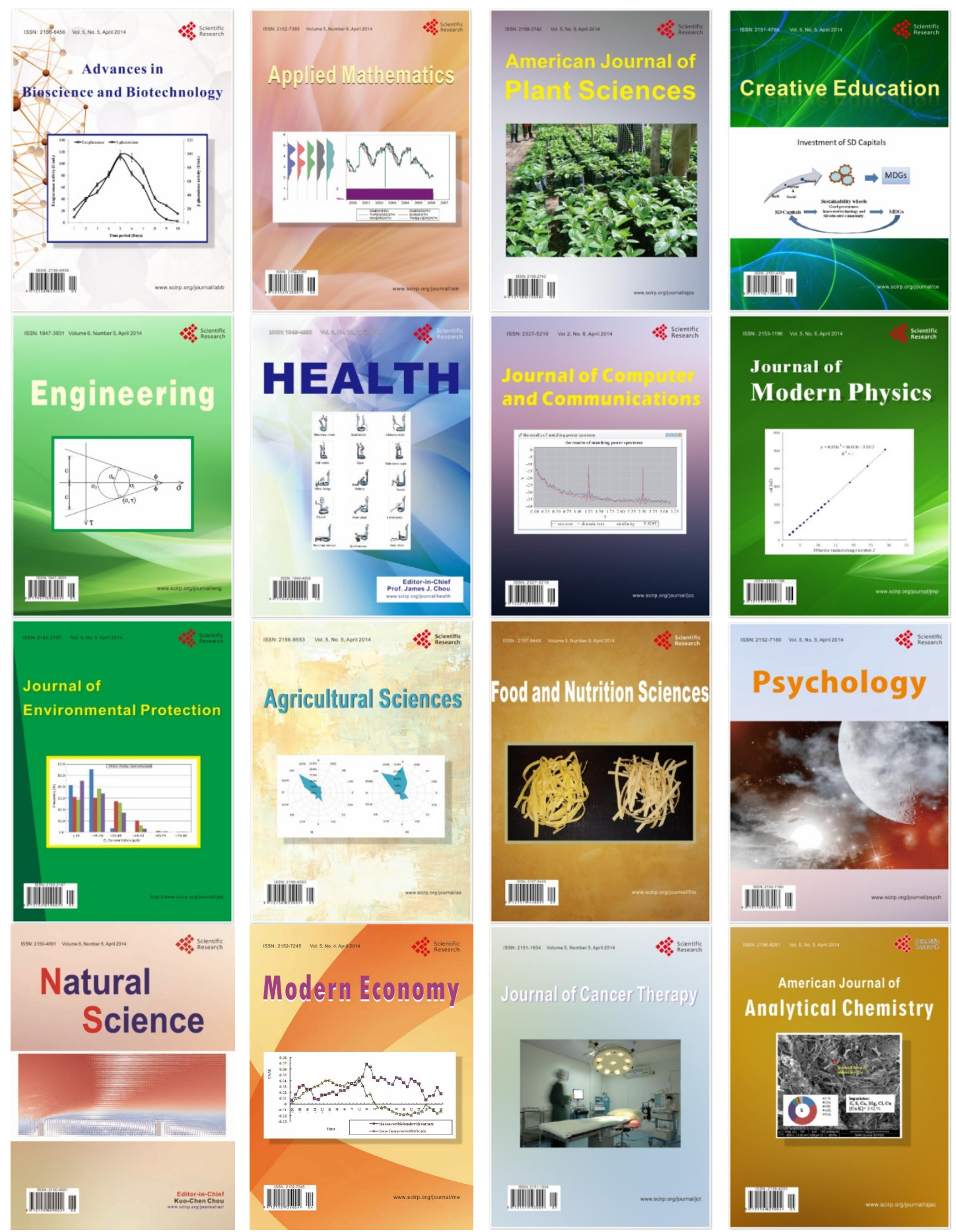